\section{The fog that was not}

Ahmed H. Zewail

n macroscopic systems, we can use classical mechanics to describe an object's motion with precise knowledge of where the object is and where it is going. But the microscopic world is governed by the laws of quantum mechanics, which rob us of this precision. We can no longer simultaneously predict the object's position and momentum, or its energy measured over a finite period of time. We accommodate this by recognizing two of the most powerful and yet indigestible concepts: the uncertainty principle and the particle-wave duality of matter.

The duality concept states that all particles, even molecules, can behave as waves. This also holds for light. In the nineteenth century, Maxwell showed that light consists of electromagnetic waves; and in 1905 Einstein described its particle nature as 'photons' - quanta of energy. The link with matter was made by de Broglie in 1924, when he showed by analogy that a particle has a wavelength associated with it by virtue of its motion.

The complementarity of these two descriptions is interwoven with the concept of coherence. Two or more waves can produce interference patterns when their amplitudes add up coherently. Interference phenomena, observed for both light and matter, embody many abstract concepts, including superposition of waves and the uncertainty principle.

For matter, superpositions analogous to those of light waves can be formed from matter wavefunctions. The Schrödinger equation yields wavefunctions together with their probability distributions, which are diffuse over position space. But if these waves are added up coherently with well-defined phases, the probability distribution becomes localized in space. The resultant wave packet and its associated de Broglie wavelength has the essential character of a classical particle: a trajectory in space and time with a welldefined (group) velocity and position - a localization is on the atomic scale. this ratio is about 1:70 for typical bonds. moving classical marble but at atomic scale!

The association of a wave character to particle motion through de Broglie's relationship is consistent with Heisenberg's mechanics, which define for all quantum systems an imprecision in position and in momentum. Similarly, there is an uncertainty relationship between time and energy. The joint effect of these two relations is the crux of the matter - short time is the way to localization. With femtosecond resolution,

With such localization, a classical picture emerges. The atomic motions in any chemical or biological transformation are dynamic, with a speed of about 1 kilometre per second. So about 100 femtoseconds are needed to record atomic-scale dynamics over a distance of an angström. Freezing these motions as reactions unfold and pass through their transition states - the configurations between reactants and products - requires resolution in space and time, and only on this timescale (femtochemistry) can we directly observe the processes of breaking and making bonds.

If this is the case, why was it thought that femtosecond timescales would not be of value? There are two reasons. First, the energy uncertainty at this timescale was considered 'huge', and it was feared that the quantized vibrational-rotational states of molecules would be 'ruined' by using femtosecond resolution. Second, wave-packet spreading in microscopic systems was thought to be severe because of intra- and intermolecular interactions. Accordingly, femtosecond resolution would be of limited use for studies in chemistry and biology. Had the energy states been prepared incoherently, this would have been true, but this is not so. What is relevant is the energy uncertainty relative to the binding energy - for a 50-femtosecond duration,

Experimentally, atomic-scale dynamics of molecules and their reactions have now been observed and studied in all phases of

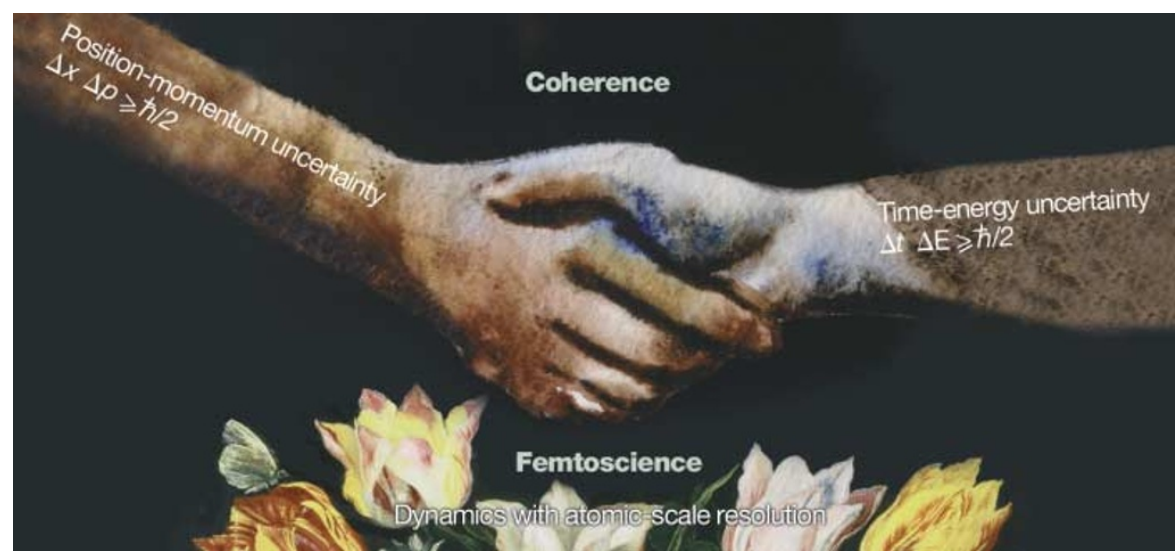

The uncertainty paradox

Femtosecond resolution is essential for observing atomic-scale dynamics in chemistry and biology. But quantum uncertainty was thought to be an obstacle - why wasn't it?

matter. One unique example is the breaking and remaking of bonds between sodium and iodine atoms. The wave packet oscillates periodically, showing particle-type behaviour during the whole reaction. The ensemble of molecules behaves in harmony, showing 'single-molecule' motion. The initial packet is indeed highly localized at about $0.1 \AA$ and remains intact for many periods of its motion. The de Broglie wavelength is also consistent with sub-angström localization. This motion conforms to the coherent superposition and probing described above. Experiments in the condensed phase and in biological systems can be described similarly.

Why is coherence robust? To see motion in real systems, localized wave packets must form in every molecule, and there must also be a limited spread in position among the wave packets formed in the millions of molecules studied. This is achieved by the welldefined initial equilibrium configuration of the molecules before excitation and by the 'instantaneous' femtosecond launching of the packet. The spatial confinement of the initial ground state, typically $0.05 \AA$, ensures that all molecules, each with its own coherence, begin their motion in a bond-distance range much smaller than that of the actual motion, typically 5-10 $\AA$. The femtosecond launching ensures that this narrow range of bond distance is maintained throughout preparation. Unless molecular and ensemble coherences are destroyed, the motion of the ensemble is that of a single-molecule trajectory.

Eugene Wigner and Edward Teller debated the uncertainty paradox for picosecond timeresolution in a lively exchange in 1972 . But, because of coherence, the uncertainty paradox is not a paradox even for femtoscience, and certainly not for the dynamics of physical, chemical and biological changes. Charles Townes encountered objections in the realization of the maser because of concern about the uncertainty principle, but coherence of photons was the key to success. As we cross the femtosecond barrier into the attosecond regime for studies of electron dynamics, we must recall this vital role of coherence; otherwise the spectre of quantum uncertainty might veil the path to new discoveries.

Ahmed H. Zewail is in the Chemistry and Physics Divisions, California Institute of Technology,

Pasadena, California 91125, USA. 